\title{
Estimation of sand suspension in a secondary channel of an alpine river
}

\author{
Benoît Camenen ${ }^{1, *}$, Guillaume Dramais ${ }^{1}$, Alexis Buffet ${ }^{1}$, Fabien Thollet $^{1}$, Chloé Le \\ Bescond $^{1}$, Mickaël Lagouy ${ }^{1}$, Céline Berni ${ }^{1}$, and Jérôme Le Coz ${ }^{1}$ \\ ${ }^{1}$ Irstea, UR RiverLy, centre de Lyon-Villeurbanne, 5 Rue de la Doua, CS 20244, F-69625 Villeurbanne \\ Cedex, France
}

\begin{abstract}
In this paper, we present some first attempt to measure graded suspension of sands in an alpine river. It is a real challenge to measure gradedsuspensions of sands in such dynamic rivers. Specific measurements of the graded suspension in the middle of a secondary channel were attempted using a sampler that allows simultaneous sampling at three different elevations $(5 \mathrm{~cm}$, $10 \mathrm{~cm}$, and $20 \mathrm{~cm}$ ) above the bed. Very large concentrations (up to $C=40 \mathrm{~g} / \mathrm{l}$ ) of fine sediments in suspension were measured during a dam reservoir flushing event. It included large amounts of sand (from 10 to $60 \%$ of the measured concentration). A substantial graded suspension of sand was measured even for relatively low velocities. Sand fluxes estimates for varying bed shear stresses during the event are discussed. In particular, based on the measured bed shear stress, a semi-empirical formula developed for sand suspension is applied to test its ability to predict sand flux in alpine rivers. The sensibility of such formula to the grain size is very high whereas large uncertainties exist in the measurement ( $d_{50}$ varies from 0.1 to $0.3 \mathrm{~mm}$ depending on the sample), which makes its application difficult at the moment.
\end{abstract}

\section{Introduction}

A substantial amount of fine sediments can be found in piedmont gravel bed rivers. It remains however very difficult to estimate the total amount of fine sediments since they are easily washed out and can also infiltrate the gravel matrix. Moreover, very different dynamics can be observed from one class of sediments to another, i.e. washload made of silts versus graded-suspension of sands. It is a real challenge to measure graded-suspensions of sands in such dynamic rivers since gravel bedload may occur at the same time. As a consequence, measurements are generally limited to washload (through surface sampling and/or turbidity measurement) or bedload of coarse particles (by using bedload sampler with a coarse net or by using tracers). Even if some recent surrogate methods exist for sand [1], there is a need of sampling to calibrate them and they are often limited to navigable rivers [2-6].

Some field experiments to measure sand suspension in a secondary channel of an alpine river are presented here. A similar protocol as Camenen et al. $(2015,2016)$ protocol $[7,8]$ was set in order to estimate bed shear stress over the gravel bar. Experimental results on the

*e-mail: benoit.camenen@irstea.fr 
graded sand suspension using automatic sampler adapted to a specific frame are discussed together with some modelling.

\section{Field measurements}

\subsection{Location of the field measurements}

The Arc en Maurienne River is a constricted alpine river characterized by a nival hydrologic regime and marked by an intense input of fine sediments from the catchment (approximately $2000 \mathrm{~km}^{2}$ ). The area of interest is a single gravel bar located within a system of alternate bars $10 \mathrm{~km}$ downstream of St-Jean-de-Maurienne (see Figure 1) with a mean slope of $0.6 \%$. This reach has been studied since 2005 [9-11]. One point of interest of this site is that flushing events of the three run-of-the-river dams (see Figure 1) are conducted yearly in June allowing the performance of in-situ experiments at a specified date known in advance. Topographic, bathymetric and flow measurements are regularly carried out during these flushing events. Bedload transport was assessed on a gravel bar using Pit-tag transponders [12] or on the main channel using a bedload sampler from a bridge [13]. A significant focus has also been made on the fine sediments dynamics during the flushing events [14-16] and the impact of the gravel bar on fine sediment exchange between the bed and the flow appeared to be a significant issue.

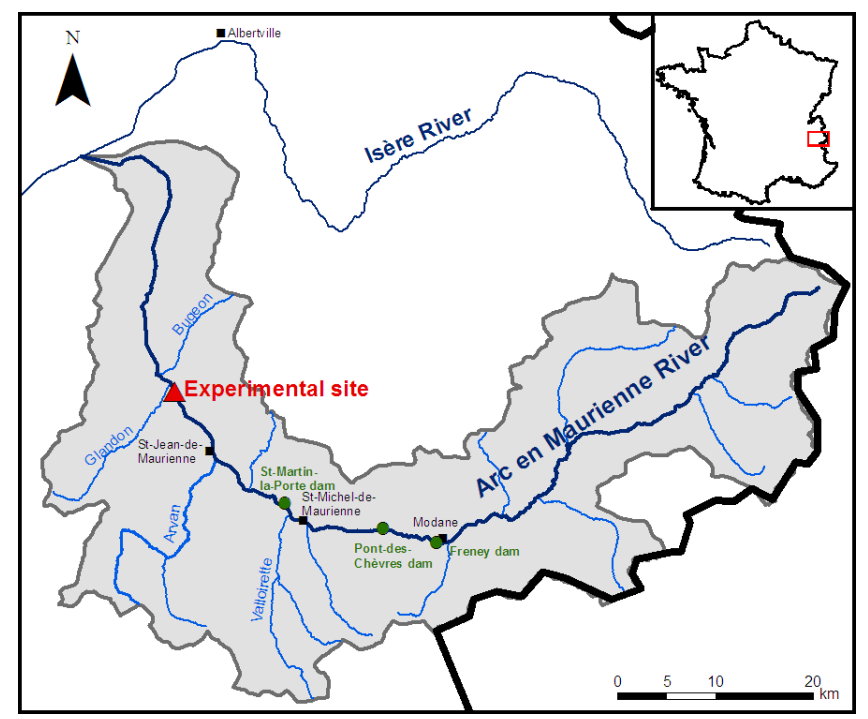

Figure 1. Location map (green points correspond to run-of-the-river dams).

\subsection{Hydro-sedimentary measurements}

Generally, a flushing event lasts for around 12 hours and follows a designed hydrograph with two plateaus, one at $Q \approx 80 \mathrm{~m}^{3} / \mathrm{s}$ and a second one at $Q \approx 130 \mathrm{~m}^{3} / \mathrm{s}$, both lasting for four hours approximately (Figure $3 \mathrm{a}$ ). The studied gravel bar is totally inundated for a flow discharge $Q \approx 100 \mathrm{~m}^{3} / \mathrm{s}$. The gravel bar was instrumented for measuring the main hydraulic characteristics (camera positioned on a mast for LSPIV -large scale particle image velocimetry, GRPs -ground reference points-, pressure gauges along the secondary channel) following 
Camenen et al. (2015, 2016) protocol [7, 8] (Fig. 2a). The field campaign included topographic measurements of the gravel bar before and after the event. LSPIV measurements were achieved during the flushing event in order to estimate surface velocities in the secondary channel and over the gravel bar when inundated. Pressure gauges were set along the secondary channel to measure both water depth and slope.

Specific measurements of the graded suspension in the middle of the secondary channel were attempted using a sampler that allows simultaneous sampling at three elevations $(5 \mathrm{~cm}$, $10 \mathrm{~cm}$, and $20 \mathrm{~cm}$ ) above the bed (Fig. 2b). Such system was inspired by the Nile sampler [17]. For this purpose, three automatic ISCO-type samplers were used and adapted to a frame (adapted from the Delft bottle frame) set in the middle of the secondary channel.

(a)

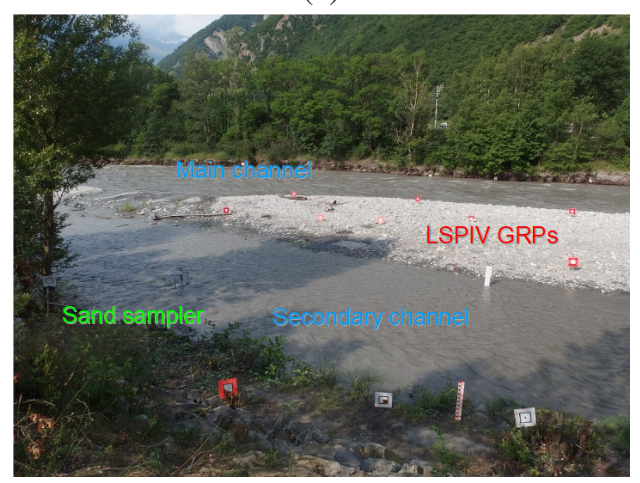

(b)

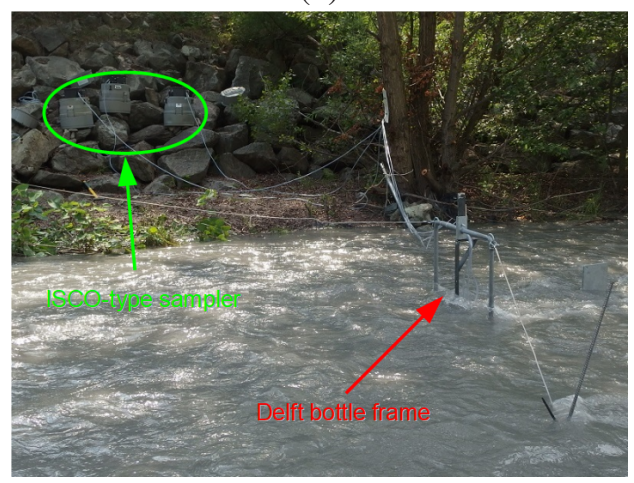

Figure 2. Photos of the experiment (a) and of the sand sampler (b).

A hydro-sedimentary station located $9 \mathrm{~km}$ upstream of the study site (at St-Jean-deMaurienne) monitored the fine sediment fluxes. Additional samplings were conducted manually during the event from a bridge ( $2 \mathrm{~km}$ downstream of the gravel bar) and next to the gravel bar on the secondary channel. Sampling results from the three positions are very consistent considering an uncertainty of $10 \%$ (see Fig. $3 \mathrm{~b}$ where concentrations excluding sand are presented). It indicates that deposition/erosion of fine sediments is negligible compared to the overall flux. During the previous flushing events, very large concentrations of fine sediments in suspension were often observed, up to $C_{\max }=30 \mathrm{~g} / 1$ [16]. In 2017, the peak concentration reached $C_{\max }=25 \mathrm{~g} / \mathrm{l}$ (Figure 3 ).

It is important to note here that sand suspension was observed even for surface sampling. In the calibration of the turbidimeter, they were however excluded to avoid bias in the estimation of the total fine sediment fluxes. Due to the sensitivity of turbidity on particle diameter and the heterogeneity in sand concentration throughout a river section, we suggest to estimate sand suspension fluxes separately from washload. In Fig. 3b, some points (squares between 2 and $4 \mathrm{pm}$ ) appeared underestimated; we believe samplings were biased because the pipe got obstructed with sands.

\subsection{Bed shear stress}

The section averaged bed shear stress was estimated in the secondary channel following the equation:

$$
\tau_{m, S}=\rho g R_{h} S
$$


(a)

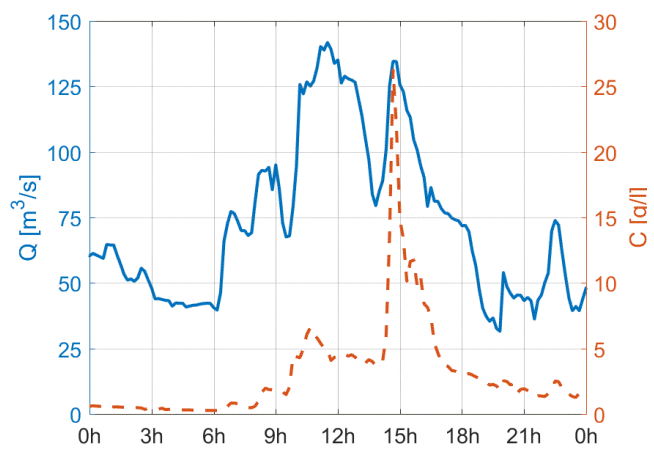

(b)

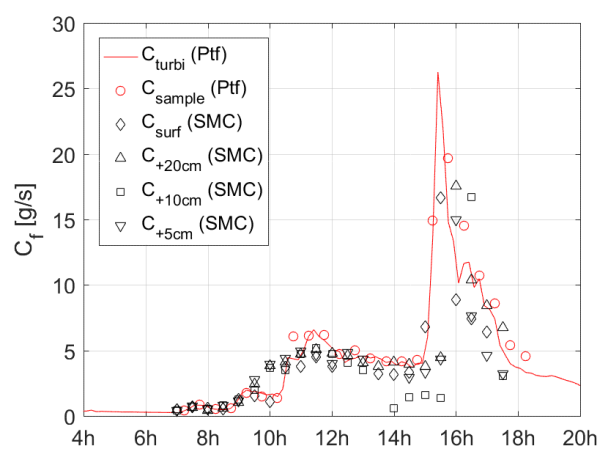

Figure 3. Discharge and concentration time series at the Pontamafrey hydro-sedimentary station during the 2017 flushing event (a) including all fine sediment samples (b). Ptf stands for Pontamafrey, SMC for Ste-Marie-de-Cuines.

where $\rho$ is the water density, $g$ the acceleration of gravity, $R_{h}$ the hydraulic radius of the secondary channel, and $S$ the water slope measured in the secondary channel. In Fig. $4, \tau_{m, S}$ is plotted as a function of time together with some LSPIV results. The calculation of the bed shear stress was estimated from the surface velocity measurements assuming a constant bed roughness. It has been computed following two methods: first by calculating bed shear stress locally and averaging them throughout the secondary channel width; and second by calculating $\tau_{m}$ using directly section-averaged parameters:

$$
\begin{aligned}
\tau_{m, U} & =\frac{1}{Y_{s c}} \int_{y=0}^{Y_{s c}} \rho\left(\frac{\kappa}{\left.1+\log \left[k_{s} / 30 h\right)\right]}\right)^{2} U^{2} d y \\
\tau_{m, \bar{U}} & =\rho\left(\frac{\kappa}{\left.1+\log \left[k_{s} / 30 \bar{h}\right)\right]}\right)^{2} \bar{U}^{2} d y
\end{aligned}
$$

where $Y_{s c}$ is the width of the secondary channel, $\kappa=0.41$ the Von Karman constant, $k_{s}=2 d_{90}$ the roughness height (where $d_{90} \approx 0.1 \mathrm{~m}$ ), $h$ and $U$ the local water depth and depth-averaged velocity estimated from LSPIV measurements, respectively, $\bar{h}$ and $\bar{U}$ their section-averaged value. If results are qualitatively consistent, a significant scatter is observed for the LSPIV results (eq. 3) compared to Eq. 1. In the following, Eq. 1 will be used since a continuous data set is obtained.

\subsection{Sand concentrations}

About 80 samples were collected during the flushing event every half-hour (four samples at $+5 \mathrm{~cm},+10 \mathrm{~cm},+20 \mathrm{~cm}$, and at the surface, respectively). Samples were analysed according to the ASTM D3977 standard to correctly estimate the sand fraction, i.e. sand fraction was first sieved and analysed separately. All the results for the graded suspension are summarized in Tab. 1 including the hydraulic conditions measured and estimated in the secondary channel. Grain size distribution (GSD) analysis was achieved on the sand fraction using a laser grain sizer (Cilas 1190). A significant scatter in the measured GSD was observed with a median grain size $d_{s}$ varying from 0.1 to $0.3 \mathrm{~mm}$. Part of this scatter may be due to the difficulty to analyse the sand fraction with the laser grain sizer (based on a small sub-sample). 


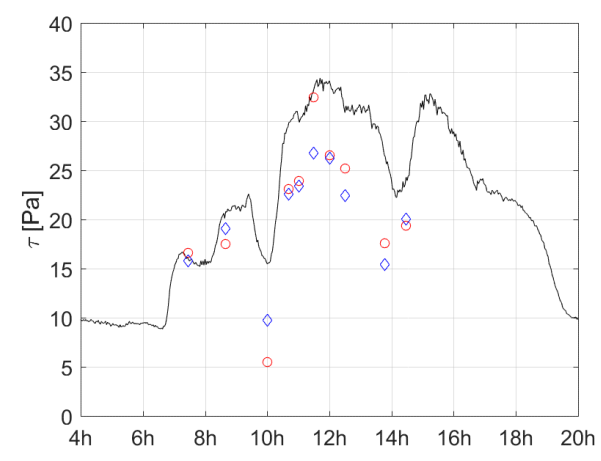

Figure 4. Bed shear stress time series at Ste-Marie-de-Cuines study site during the 2017 flushing event. Blue and red circles correspond to LSPIV results using Eqs. 2 and 3, respectively.

Table 1. Experimental conditions and results for the graded suspension measurements during the 2017 flushing event (1st June). $z_{w}$ is the water surface elevation of the secondary channel near the sampler, $Q_{s c}$ and $V$ are the discharge and section-averaged velocity in the secondary channel, respectively, measured thanks to LSPIV measurements.

\begin{tabular}{llllllllll}
\hline time & $\begin{array}{l}z_{w} \\
(\mathrm{~m} \mathrm{NGF})\end{array}$ & $\begin{array}{l}Q_{A r c} \\
\mathrm{~m}^{3} / s\end{array}$ & $\begin{array}{l}Q_{s c} \\
\mathrm{~m}^{3} / s\end{array}$ & $\begin{array}{l}V \\
\mathrm{~m} / \mathrm{s}\end{array}$ & $\begin{array}{l}\tau_{m, S} \\
\mathrm{~Pa}\end{array}$ & $\begin{array}{l}C_{s,+5 c m} \\
\mathrm{~g} / \mathrm{L}\end{array}$ & $\begin{array}{l}C_{s,+10 c m} \\
\mathrm{~g} / \mathrm{L}\end{array}$ & $\begin{array}{l}C_{s,+20 c m} \mathrm{~g} / \mathrm{L} \\
C_{s, \text { surf }}\end{array}$ \\
\hline $07 \mathrm{~h} 00$ & 455.14 & 43 & - & - & 15.0 & 0.17 & 0.19 & 0.10 & 0.22 \\
$07 \mathrm{~h} 30$ & 455.15 & 75 & 1.40 & 0.69 & 15.8 & 0.57 & 0.56 & 0.69 & 0.22 \\
$08 \mathrm{~h} 00$ & 455.16 & 72 & - & - & 16.0 & 0.55 & 0.53 & 0.40 & 0.18 \\
$08 \mathrm{~h} 30$ & 455.22 & 69 & 2.80 & 0.88 & 19.8 & 1.3 & 1.2 & 1.1 & 0.13 \\
$09 \mathrm{~h} 00$ & 455.23 & 92 & - & - & 21.0 & 0.61 & 0.75 & 0.70 & 0.37 \\
$09 \mathrm{~h} 30$ & 455.23 & 90 & - & - & 21.1 & 1.8 & 2.8 & 1.9 & 0.45 \\
$10 \mathrm{~h} 00$ & 455.13 & 79 & 1.00 & 0.53 & 15.5 & 5.0 & 6.2 & 5.0 & 0.25 \\
$10 \mathrm{~h} 30$ & 455.34 & 73 & 5.90 & 1.03 & 27.8 & 7.1 & 9.5 & 7.7 & 1.7 \\
$11 \mathrm{~h} 00$ & 455.38 & 124 & 6.00 & 1.06 & 30.4 & 5.6 & 7.4 & 7.4 & 3.6 \\
$11 \mathrm{~h} 30$ & 455.44 & 126 & 8.50 & 1.16 & 33.3 & 7.3 & 11.0 & 8.6 & 2.6 \\
$12 \mathrm{~h} 00$ & 455.44 & 139 & 8.70 & 1.16 & 34.1 & 7.8 & 14.9 & 8.1 & 2.6 \\
$12 \mathrm{~h} 30$ & 455.41 & 136 & 7.40 & 1.04 & 30.9 & 7.3 & 13.7 & 7.5 & - \\
$13 \mathrm{~h} 00$ & 455.40 & 127 & - & - & 31.5 & 7.7 & 31.7 & 5.0 & - \\
$13 \mathrm{~h} 30$ & 455.38 & 127 & - & - & 29.2 & - & - & 5.0 & 1.8 \\
$14 \mathrm{~h} 00$ & 455.31 & 110 & 4.90 & 0.85 & 23.9 & - & 0.16 & 5.7 & 1.7 \\
$14 \mathrm{~h} 30$ & 455.31 & 81 & 4.00 & 0.91 & 23.9 & 6.0 & 22.3 & 3.4 & 1.3 \\
$15 \mathrm{~h} 00$ & 455.43 & 95 & - & - & 32.1 & 5.2 & 16.3 & 4.4 & 2.6 \\
$15 \mathrm{~h} 30$ & 455.45 & 134 & - & - & 31.5 & 9.6 & 14.0 & 6.4 & 6.8 \\
$16 \mathrm{~h} 00$ & 455.40 & 119 & - & - & 28.9 & 13.8 & - & 13.8 & 4.6 \\
$16 \mathrm{~h} 30$ & 455.35 & 102 & - & - & 25.2 & 11.0 & - & 11.7 & 5.2 \\
$17 \mathrm{~h} 00$ & 455.32 & 85 & - & - & 23.6 & 3.9 & - & 7.2 & 4.6 \\
$17 \mathrm{~h} 30$ & 455.30 & 81 & - & - & 22.6 & 3.8 & - & 6.5 & - \\
\hline
\end{tabular}

Sand concentration measurements are plotted in Fig. 5a as a function of the vertical position (each coloured symbols correspond to one of the 22 experimental conditions). One can observe a large variability of the sand concentrations during the event but a moderate vertical gradient. As a consequence, one can conclude that the sediment diffusivity did not vary significantly during the event (i.e. for bed shear stress varying from 15 to $35 \mathrm{~Pa}$ ). On the other 
hand, the bed concentration $\left(C_{z=+5 \mathrm{~cm}}\right)$ varied by two orders of magnitude and so is much more sensitive to the bed shear stress and/or to the sand availability.

(a)

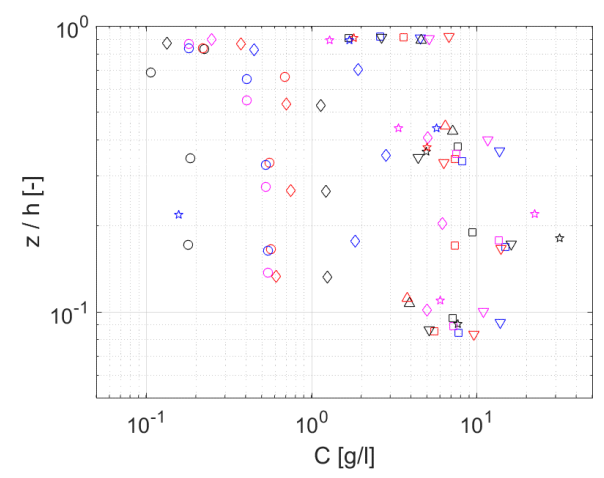

(b)

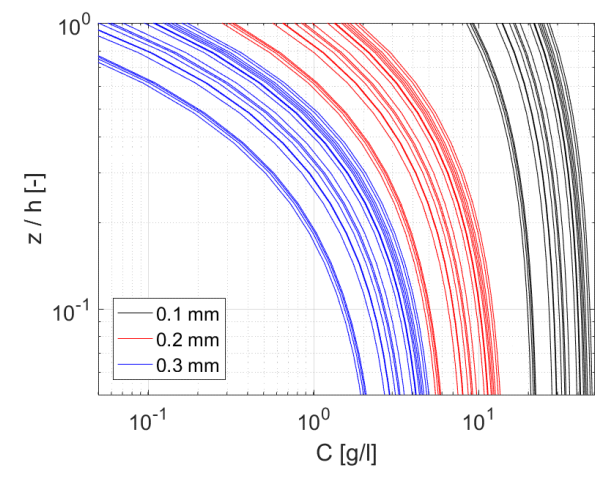

Figure 5. Measured (a) and modelled (b) sand concentration profiles along the flushing event using different hypothesis on the grain size. The model was applied for three different grain size on the 22 different hydraulic conditions.

\section{Model and results}

\subsection{Model}

The sand concentrations were estimated using the Camenen \& Larson (2008) [18] equations. For a steady current, it presents a exponential profile since the sediment diffusivity $\epsilon_{s}$ is assumed constant over the water depth :

$$
C(z)=C_{R} \exp \left(-\frac{W_{s}}{\epsilon_{s}} z\right)
$$

where $W_{s}$ is the settling velocity of the sand particles of diameter $d_{s}, C_{R}$ a reference concentration expressed as follows :

$$
C_{R}=1.5 \times 10^{-3} \rho_{s} \exp \left(-0.2 D_{s}\right) \theta \exp \left(-4.5 \frac{\theta_{c r}}{\theta}\right)
$$

with $D_{s}=\left[g\left(\rho_{s} / \rho-1\right) / v^{2}\right]^{1 / 3} d_{s}$ the dimensionless grain size $\left(\rho_{s}\right.$ : sediment density; $v$ : kinematic viscosity), $\theta=\tau /\left[\left(\rho_{s}-\rho\right)\right]$ the Shields number and $\theta_{c r}$ its critical value for inception of movement. The sediment diffusivity is calculated as follows :

$$
\epsilon_{s}=\frac{1}{6} \kappa u_{*} h
$$

where $\kappa=0.41$ is the Von Karman constant, $u_{*}=(\tau / \rho)^{1 / 2}$ the friction velocity and $h$ the mean water depth. 


\subsection{Results}

In Fig. 5b, we plotted vertical concentration profiles obtained from the model using three different grain sizes $\left(d_{s}=0.1,0.2,0.3 \mathrm{~mm}\right)$ for the 22 different hydraulic conditions (estimated in a same way as for Fig. 5a). It appears that the model cannot reproduce the scatter in experimental data by using a single grain size, but it is very sensitive to the grain size as it could be expected from the settling velocity dependency. The model provides however an estimate of the concentrations (same order of magnitude and similar vertical gradients).

We tried to estimate what kind of bed mixture would lead to such concentration profiles. Assuming four classes of sediment composing the bed, three of sand (with grain diameters of $0.1,0.2$, and $0.3 \mathrm{~mm}$ respectively) and one class of gravels, the modelled concentration profiles for the assumed bed mixture can be calculated following:

$$
C_{s}=\Sigma_{i=1}^{3} \alpha_{i} C_{i}
$$

where $i$ stands for the three classes of sand. Using a try and error method, we eventually found $\alpha_{0.1 \mathrm{~mm}}=2 \%, \alpha_{0.2 \mathrm{~mm}}=40 \%, \alpha_{0.3 \mathrm{~mm}}=40 \%$, and $\alpha_{\text {gravel }}=18 \%$. Such choice leads to the results presented in Fig. 6a. It is interesting to note that the finest class can easily overwhelm the final concentration. The grain size repartition at the four levels is plotted in Fig. $6 \mathrm{~b}$ based on the model results. Even if the bed mixture is assumed to be composed of $2 \%$ of fine sand $\left(d_{s}=0.1 \mathrm{~mm}\right)$, this class represents between 35 and $50 \%$ of the concentration close to the surface. On the opposite, the coarse sand $\left(d_{s}=0.3 \mathrm{~mm}\right)$ represents less than $20 \%$ of the concentration at $z=+5 \mathrm{~cm}$ and less than $5 \%$ close to the surface.

(a)

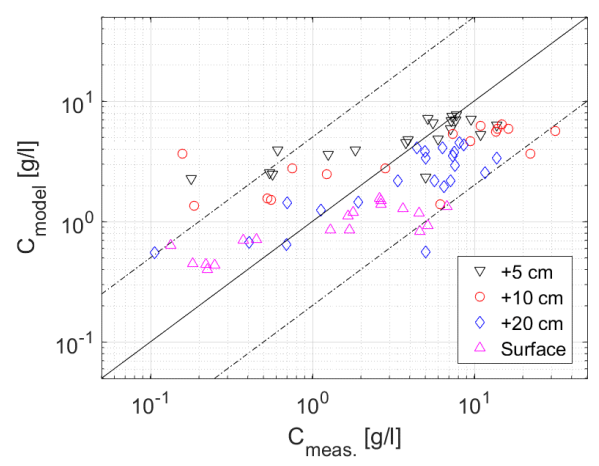

(b)

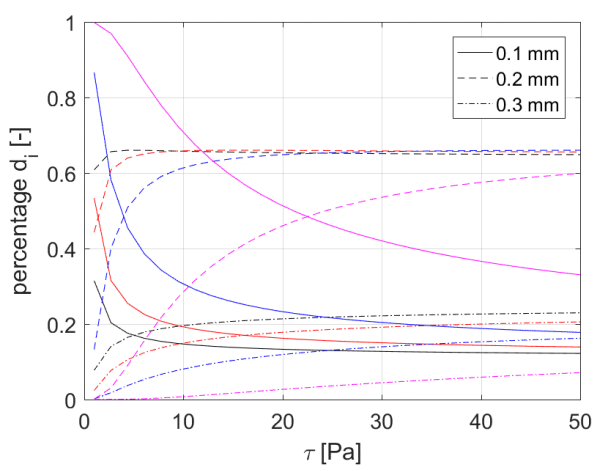

Figure 6. Comparison between modelled and measured sand concentration assuming a sand mixture (a) and resulting grain size repartition at the four levels (black: $+5 \mathrm{~cm}$, red: $+10 \mathrm{~cm}$, blue: $+20 \mathrm{~cm}$, magenta: surface) as a function of the estimated bed shear stresses (b).

\section{Conclusion}

Results of a first attempt to measure sand suspension in a alpine river (Arc, France) were presented showing that large concentrations of sand can be observed in such rivers. Measurements were achieved using three automatic samplers pumping at three different elevations over the river bed thanks to a structure supporting the pipe entrance. Such system may be improved by using more powerful pumps and/or larger pipes in order to avoid sand obstruction 
of the pipe. It appeared also fundamental to focus more carefully on the grain size distribution of the different samples. Indeed, the Camenen \& Larson (2008) [18] model indicates that graded suspension of sand is very sensitive to the grain size and a vertical gradient in the grain size (coarse close to the bed) can easily be observed.

\section{Acknowledgements}

This study was supported by Irstea and EDF-CIH.

\section{References}

[1] J.R. Gray, J.W. Gartner, Technological advances in suspended-sediment surrogate monitoring, Water Resources Research, 45, 1 (2009)

[2] L.C. van Rijn, Manual sediment transport measurements in rivers, estuaries and coastal seas (Aqua publication, Blokzijl, The Netherland, 2007), $500 \mathrm{p}$.

[3] C.L. Amos et al., The measurement of sand transport in two inlets of Venice lagoon, Italy, Estuarine, Coastal \& Shelf Science 87, 225 (2010)

[4] E. Armijos et al., Measuring and modeling vertical gradients in suspended sediments in the Solimões/Amazon River, Hydrological Processes 31, 654 (2017)

[5] A.B. Gitto et al., Representative point-integrated suspended sediment sampling in rivers, Water Resources Research, 53, 2956 (2017)

[6] A.W.M. Pomeroy et al., Sediment transport in the presence of large reef bottom roughnes, Journal of Geophysical Research: Oceans 122, 1 (2017)

[7] B. Camenen et al., Field experiment on the dynamics of fine and coarse sediments over a gravel bar in an alpine river, in Proc. 36th IAHR Congress (The Hague, The Netherlands, 2015)

[8] B. Camenen et al., Estimation of the volume of a fine sediment deposit over a gravel bar during a flushing event, in River Flow (St Louis, Missouri, USA, 2016)

[9] M. Jodeau, Ph.D. thesis, Claude Bernard University, Lyon, France (2007), 205p. (in French)

[10] M. Jaballah, Ph.D. thesis, Claude Bernard University, Lyon, France (2013), 196 p.

[11] M. Jaballah et al., Alternate bar development in an alpine river following engineering works, Advances in Water Resources 81, 103-113 (2015)

[12] B. Camenen et al., An estimation of gravel mobility over an alpine river gravel bar (Arc en Maurienne, France) using PIT-tag tracers, in River Flow (Braunschweig, Germany, 2010), pp. 953-960

[13] B. Camenen et al., Tentative measurements of bedload transport in an energetic alpine gravel bed river, in River Flow (San Jose, Costa Rica, 2012), pp. 379-386

[14] B. Camenen et al., Conceptual modelling of the sediment flux during a flushing event (Arc en Maurienne, France), in Proc. 8th ICHE (Nagoya, Japan, 2008), CD Rom

[15] G. Antoine et al., Estimation des flux de matières en suspension lors des chasses hydrauliques de l'Arc de 2006 à 2011, La Houille Blanche 4, 43-49 (2013), (in French)

[16] G. Antoine, Ph.D. thesis, Grenoble University, France (2013), 282 p. (in French)

[17] L.C. van Rijn, M. Gaweesh, A new total load sampler, Journal of Hydraulic Engineering 118, 1686 (1992)

[18] B. Camenen, M. Larson, A General Formula for Noncohesive Suspended Sediment Transport, Journal of Coastal Research 24, 615-627 (2008) 